\title{
Atuação de uma equipe interprofissional no Programa Saúde na Escola: Relato de experiência
}

\author{
Performance of an interprofessional team in the School Health Program: Experience report \\ Desempeño de un equipo interprofesional en el Programa de Salud Escolar: Informe de experiencia
}

Recebido: 07/03/2021 | Revisado: 12/03/2021 | Aceito: 19/03/2021 | Publicado: 25/03/2021

Carlos Rafael Lopes de Azevedo

ORCID: https://orcid.org/0000-0002-5756-7863

Universidade Federal do Amazonas, Brasil

E-mail: crafazevedo@gmail.com

Lorrayne Caroline Pereira Ferreira

ORCID: https://orcid.org/0000-0002-5358-6055

Universidade Federal do Amazonas, Brasil

E-mail: lorrayne.pf@gmail.com

Milene Maciel Rodrigues

ORCID: https://orcid.org/0000-0002-4175-5595

Universidade Federal do Amazonas, Brasil

E-mail: lorrayne.pf@gmail.com

Juliana Vieira Saraiva

ORCID: https://orcid.org/0000-0001-9240-0981

Universidade Federal do Amazonas, Brasil

E-mail: julianasaraiva73@gmail.com

Carina Toda

ORCID: https://orcid.org/0000-0003-1709-0877

Universidade Federal do Amazonas, Brasil

E-mail: carinatoda@yahoo.com.br

Sineide Santos de Souza

ORCID: https://orcid.org/0000-0001-5934-5271

Universidade Federal do Amazonas, Brasil

E-mail: sineidesantosdesouza@gmail.com

Janete Maria Rebelo Vieira

ORCID: https://orcid.org/0000-0002-7125-9082

Universidade Federal do Amazonas, Brasil

E-mail: rebelovieirajm@gmail.com

Miele Gonçalves de Mesquita

ORCID: https://orcid.org/0000-0001-8881-9376

Secretaria Municipal de Saúde de Manaus, Brasil

E-mail: mielemesquita@yahoo.com.br

Sandra Maria Rodrigues Rubens

ORCID: https://orcid.org/0000-0002-8764-0427

Secretaria Municipal de Saúde de Manaus, Brasil

E-mail: rubens.sandra@hotmail.com

Sthefane Fontes Ponce de Leon

ORCID: https://orcid.org/0000-0001-6245-193X

Secretaria Municipal de Saúde de Manaus, Brasil

E-mail: leonsther@gmail.com

Laneide Stella de Jesus Lima Vieira

ORCID: https://orcid.org/0000-0003-4394-7408

Secretaria Municipal de Saúde de Manaus, Brasil

E-mail: laneide.lima@pmm.am.gov.br

\section{Resumo}

O presente trabalho visa relatar a vivência de uma equipe interprofissional na implementação de ações de educação em saúde para promoção da alimentação saudável entre escolares da rede pública de ensino, através do Programa Saúde na Escola (PSE), uma das políticas desenvolvidas pelo Ministério da Saúde (MS). Trata-se de um estudo qualitativo, descritivo, do tipo relato de experiência. As ações foram realizadas entre os meses de julho a agosto de 2019 em uma escola municipal com crianças do $1^{\circ}$ ao $5^{\circ}$ ano do ensino fundamental I, durante um período de 04 dias, totalizando 22 turmas do turno matutino e vespertino, na cidade de Manaus/AM. Em todo o processo ficou evidente para a equipe a importância da interação interprofissional explícita entre acadêmicos, docentes e profissionais de saúde, o que tornou possível estabelecer práticas colaborativas entre a profissões envolvidas. Foi possível vivenciar 
um dos lemas da Educação Interprofissional em Saúde (EIP) “aprender juntos para trabalhar juntos através de práticas colaborativas em saúde".

Palavras-chave: Educação interprofissional; Educação em saúde; Prática colaborativa; Atenção à saúde.

\begin{abstract}
The present work aims to report the experience of an interprofessional team in the implementation of health education actions to promote healthy eating among public school students, through the Programa Saúde na Escola (PSE), one of the policies developed by the Ministry of Health (MS). This is a qualitative, descriptive, experience report type study. The actions were carried out between the months of July and August 2019 in a municipal school with children from the 1st to the 5th year of elementary school I, during a period of 04 days, totaling 22 classes of the morning and afternoon shift, in the city of Manaus / AM. Throughout the process, it was evident to the team the importance of explicit interprofessional interaction between academics, teachers and health professionals, which made it possible to establish collaborative practices between the professions involved. It was possible to experience one of the mottos of Interprofessional Education in Health (IPE) "learn together to work together through collaborative health practices".

Keywords: Interprofessional education; Health education; Collaborative practice; Health care.
\end{abstract}

\title{
Resumen
}

El presente trabajo tiene como objetivo reportar la experiencia de un equipo interprofesional en la implementación de acciones de educación en salud para promover la alimentación saludable entre los estudiantes de escuelas públicas, a través del Programa Saúde na Escola (PSE), una de las políticas desarrolladas por el Ministerio de Salud (MS). Este es un estudio de tipo informe de experiencia, cualitativo y descriptivo. Las acciones se llevaron a cabo entre los meses de julio y agosto de 2019 en un colegio municipal con niños de $1^{\circ}$ a $5^{\circ}$ año de la escuela primaria I, durante un período de 04 días, totalizando 22 clases del turno de mañana y tarde, en el ciudad de Manaus / AM. En todo el proceso, fue evidente para el equipo la importancia de la interacción interprofesional explícita entre académicos, docentes y profesionales de la salud, lo que permitió establecer prácticas colaborativas entre las profesiones involucradas. Fue posible experimentar uno de los lemas de la Educación Interprofesional en Salud (EIP) "aprender juntos a trabajar juntos a través de prácticas de salud colaborativas".

Palabras clave: Educación interprofesional; Educación en salud; Práctica colaborativa; Atención a la salud.

\section{Introdução}

A Educação Interprofissional (EIP) é uma proposta discutida, aproximadamente, desde a década de 80, nos países europeus e nos Estados Unidos. Pode ser considerada como uma crítica à lógica tradicional de formação em saúde, onde as profissões aprendem juntas sobre o trabalho a ser desenvolvido em conjunto e sobre as especificidades de cada uma profissão, com objetivo de melhorar a colaboração e a qualidade dos cuidados (Barr, 1998; 2005; Batista, 2012). Consiste em um processo de aprendizagem que prepara os profissionais através de educação interdisciplinar e das diversas experiências da realidade do trabalho em saúde, em colaboração com as comunidades para atender às necessidades multifacetadas de crianças, jovens e famílias (IPEC, 2011).

Estudos apontam que a Atenção Primária à Saúde (APS) é o nível de atenção do SUS que mais avançou em relação à prática colaborativa e trabalho em equipe, por meio de práticas integrais e com direcionamento às necessidades individuais e coletivas de uma população (Peduzzi \& Agreli, 2018). Provavelmente, tal fato seja em virtude do princípio da interprofissionalidade ser critério fundamental na orientação das equipes multiprofissionais na Estratégia Saúde da Família (ESF) (Ellery, 2014).

Dentre as políticas públicas voltadas à promoção da saúde desenvolvidas no Brasil, o Programa Saúde na Escola (PSE), instituído em 2007, é uma política intersetorial criada pelo Ministério da Saúde (MS) e Ministério da Educação (MEC) com objetivo contribuir para a formação integral dos estudantes por meio de ações de promoção, prevenção e atenção à saúde, com vistas ao enfrentamento das vulnerabilidades que comprometem o pleno desenvolvimento de crianças, adolescentes, jovens e adultos da rede pública de ensino (Ministério da Saúde, 2011).

O PSE é uma oportunidade para estimular a interatividade interprofissional no campo da prática. Esse campo é um cenário diversificado para trabalhar a aprendizagem interprofissional, pois de acordo com Colet (2002), conforme citado por Ellery et al. (2013) "a interprofissionalidade resulta do processo de integração de saberes, que se processa no campo 
epistemológico, e da colaboração interprofissional, que tem lugar na organização do trabalho, ou seja, no campo das práticas". para a prática de promoção da saúde e de prevenção de agravos à saúde e de doenças (Ministério da Saúde, 2011).

Neste contexto, com a finalidade de trabalhar a interdisciplinaridade e promover a integração ensino-serviçocomunidade, a equipe do Programa de Educação pelo Trabalho para a Saúde (PET-Saúde) Interprofissionalidade e a equipe de saúde da ESF desenvolveram atividades educativas na escola por meio do PSE sobre o tema alimentação saudável, tema sugerido pela direção da escola municipal por ser uma necessidade identificada pelos profissionais da escola.

No Brasil, o PET-Saúde constitui-se como estratégia para impulsionar a interprofissionalidade nos cursos de graduação em saúde, promover a integração ensino-serviço-comunidade no Sistema Único de Saúde (SUS), induzir mudanças nos Projetos Políticos Pedagógicos (PPC) dos cursos e desenvolver a docência e a preceptoria na saúde (França et al., 2018).

Dessa forma, o objetivo deste trabalho é relatar a vivência de uma equipe interprofissional na implementação de ações de educação em saúde para promoção da alimentação saudável entre escolares da rede pública de ensino.

\section{Metodologia}

Trata-se de um estudo qualitativo, descritivo, do tipo relato de experiência, a partir de práticas de educação em saúde vivenciadas por discentes, docentes e profissionais de saúde em uma escola pública municipal localizada na cidade de Manaus, Amazonas.

O relato de experiência aproxima a prática da teoria, descreve e contextualiza a experiência, bem como analisa o aprendizado nas pessoas envolvidas no fenômeno (Barros \& Lehfeld, 2000). A abordagem qualitativa ocupa-se com o universo de significados, motivos, aspirações, crenças, valores e atitudes, isto é, da realidade social, na qual considera-se não apenas o agir, mas a reflexão e a interpretação de ações a partir da vivência compartilhada com os pares (Minayo, 2012).

Para isso realizou-se o registro sistemático da experiência por meio da observação das atividades realizadas com vistas a desenvolver a interprofissionalidade, bem como pesquisa bibliográfica para fundamentação teórica, reflexão e discussão das atividades desenvolvidas.

A equipe PET-Saúde Interprofissionalidade compreende discentes, docentes da Universidade Federal do Amazonas (UFAM) e profissionais da Secretaria Municipal de Saúde (SEMSA) na cidade de Manaus - AM, das áreas de Odontologia, Enfermagem, Fisioterapia, Medicina e Sociologia. Entre os objetivos do PET, está o desenvolvimento de estratégias de fortalecimento da EIP, principalmente quanto à prática colaborativa, sob a perspectiva interdisciplinar com vistas a qualificar o trabalho em saúde e oferecer assistência centrada na pessoa e sua família com todas as suas peculiaridades, em busca da integralidade do cuidado (Almeida, Teston \& Medeiros, 2019).

As ações educativas foram realizadas entre os meses de julho a agosto de 2019 em uma escola pública municipal com crianças de seis a onze anos de idade do $1^{\circ}$ ao $5^{\circ}$ ano do ensino fundamental I, durante um período de 04 dias, totalizando 22 turmas do turno matutino e vespertino e tiveram o objetivo de reforçar a importância da alimentação saudável para a saúde, crescimento e desenvolvimento.

O eixo temático que norteou a atividade educativa foi baseado no componente II do manual do PSE "Promoção da Saúde e Prevenção" (Ministério da Saúde, 2011), com enfoque na realização de atividades educativas sobre a alimentação e modos de vida saudáveis com a comunidade escolar, valorizando os alimentos regionais.

O tema escolhido foi sugestão da gestora da escola e estava em acordo com projeto político-pedagógico, conforme preconiza o PSE, onde qualquer intervenção de saúde, inclusive a educativa, deve também ser pedagógica (Ministério da Saúde, 2011). Neste sentido, procuramos agendar previamente a atividade para que os professores e estudantes fossem preparados, trabalhando em um contexto interdisciplinar nas disciplinas ofertadas. 
$\mathrm{O}$ uso de metodologias ativas teve como finalidade relacionar as orientações realizadas na sala de aula com as dinâmicas práticas e lúdicas, as quais compreenderam: jogo da memória com os alimentos regionais e de custo acessível; trilha sobre a alimentação saudável; placa de sinalização de trânsito associando as cores vermelha, amarela e verde com os alimentos saudáveis e não saudáveis e a dinâmica da bandeira do Brasil, na qual os estudantes teriam que associar as cores verde, amarela e azul com os grupos de alimentos.

Cada turma era dividida em grupos para participar das atividades; que foram desenvolvidas em forma de estações, ou seja, cada grupo de alunos ia percorrendo as quatro estações de atividades, até completar a totalidade das estações.

\section{Resultados e Discussão}

A escolha do tema visou a prática do trabalho interprofissional entre as áreas de Enfermagem, Odontologia, Fisioterapia, Medicina e Sociologia para atender a demanda solicitada pela escola.

As atividades educativas compreenderam orientações gerais aos escolares sobre o consumo e a importância dos alimentos saudáveis para a saúde, enfatizando de forma lúdica, a importância da ingestão dos macronutrientes e micronutrientes e o seu benefício tanto para a saúde geral quanto para a saúde bucal. Foi incentivado o consumo de alimentos naturais, regionais e de baixo custo, devido a escola e os alunos estarem inseridos em um contexto de vulnerabilidade social.

O uso de metodologias ativas nas atividades mostrou-se desafiadora para a equipe, porém necessária para a aprendizagem, haja vista que o uso do lúdico favoreceu a participação nas atividades e o interesse na temática. Para Berbel (2011), as metodologias ativas fundamentam-se em formas de desenvolver a aprendizagem, empregando experiências reais ou simuladas, com a finalidade proporcionar a resolução adequada de problemas e/ou desafios que surgem das atividades essenciais da prática social, em diferentes contextos.

Os objetivos traçados pela equipe interprofissional no planejamento foram alcançados. Ressalta-se que o planejamento e execução das atividades educativas e lúdicas exigiu a participação ativa da equipe, sendo necessário discussão, colaboração e responsabilidade de cada membro. Acrescenta-se a necessidade de considerar diferenças socioculturais, haja vista ser uma escola inserida em um contexto de vulnerabilidade social e com considerável quantidade de crianças imigrantes da Venezuela e Haiti. Nesse sentido foi possível contribuir para o fortalecimento da autonomia dos escolares no que diz respeito a alimentação saudável.

Em todo o processo ficou evidente para a equipe PET-Saúde a importância da comunicação interprofissional entre os participantes, o que tornou possível vivenciar a colaboração eficaz. A discussão e troca de experiência entre as diferentes categorias profissionais da área de saúde durante o planejamento e implementação das atividades possibilitou o conhecimento dos papéis dos profissionais, bem como as similaridades e complementaridade entre os profissionais da equipe de saúde, o que possibilitou vivenciar a prática colaborativa e sua importância para atender as necessidades do usuário de forma integral. Dessa forma, a divisão de tarefas foi realizada de forma compartilhada, valorizando os saberes de cada profissão com vistas a evitar a duplicidade de informações repassadas aos escolares, bem como a sobrecarga dos membros da equipe.

A vivência no PET-Saúde/Interprofissionalidade estimulou a adoção de estratégias educacionais alicerçadas na EIP e promoveu as práticas colaborativas conforme visualizado por Almeida, Teston \& Medeiros (2019).

No olhar específico da enfermagem e medicina observou-se o exame físico, especialmente a inspeção, verificando a palidez mucocutânea de conjuntiva, cavidade bucal e mãos. Além disso, alterações visuais e cutâneas foram observadas com o objetivo de evidenciar alguma deficiência nutricional, e nesse sentido, encaminhar para a unidade de saúde de referência para investigação mais detalhada. 
$\mathrm{Na}$ ótica da odontologia tangenciaram na instrução em relação a interação entre uma boa alimentação e a manutenção da saúde bucal, orientações sobre uma boa escovação e a sua importância após as refeições e antes de dormir, bem como a relação entre açúcar e cárie dentária.

A fisioterapia ressaltou a importância da alimentação saudável para o fornecimento de energia ao corpo e sobre a necessidade praticar exercícios. A sociologia destacou a necessidade de considerar as crenças, valores e significados que as crianças dão a alimentação nas atividades.

Reflexões importantes e necessárias despontaram nos membros da equipe, os acadêmicos refletiram sobre a formação acadêmica e fizeram questionamentos como "estamos sendo formados para trabalhar em equipe?", apesar das iniciativas para promover o trabalho em equipe, estas ainda são incipientes. Entretanto, a vivência foi fundamental para a construção da identidade profissional dos acadêmicos. Para Santos (2005), a cultura profissional é determinada pela interação social entre todos os atores do campo profissional, desenvolvendo assim uma identidade própria do seu grupo profissional, parte importante que se constrói com a experiência.

A EIP possibilita aos estudantes aprendizado em conjunto com outros profissionais, o que contribui para o desenvolvimento de atributos e competências necessárias para o trabalho em equipe. Tal fato tem estimulado investimento em EIP na expectativa de formar profissionais de saúde com habilidades e conhecimentos para trabalhar efetivamente com outros profissionais no sistema de saúde (Reeves, 2016).

Entretanto, para o fortalecimento da EIP é crucial o apoio organizacional para solucionar os problemas relacionados à logística e recursos necessários a EIP, para aprimorar docentes e construir uma cultura comprometida com a EIP que favoreça a troca de saberes entre os atores do cenário de atenção à saúde. Além de encorajamento e apoio a estudantes e profissionais em programas de EIP pela liderança de escolas, associações e organizações envolvidas no desenvolvimento da EIP (Reeves, 2016).

Para os profissionais de saúde da unidade, a experiência favoreceu a reflexão crítica a respeito da necessidade de repensar as ações de educação em saúde, não somente para o PSE, mas também em outros programas desenvolvidos. Além de incorporar metodologias ativas, evitando a educação tradicional e verticalizada, incorporando articulação entre as diferentes expertises que a unidade pode proporcionar ao usuário.

Do ponto de vista dos docentes da universidade, ficou evidente o desafio e necessidade de incorporar as discussões sobre EIP no Núcleo Docente Estruturante (NDC), com o objetivo de inserir orientações para a mudança do modelo de formação de competências e habilidades para o efetivo trabalho em equipe dos futuros profissionais de saúde.

Estudo realizado por Peduzzi et al. (2013), considerando o cenário da formação dos profissionais de saúde no Brasil identificaram três tipos de formação, a saber: uniprofissional, multiprofissional e interprofissional, com predominância da formação uniprofissional, a qual os estudantes de uma mesma profissão aprendem de forma isolada, sem interação com estudantes de outras profissões, e concluem que a EIP, sustentáculo para a prática colaborativa, ainda está limitada a iniciativas recentes.

Observa-se a necessidade de investimento da EIP na formação com vistas a prática cada vez mais colaborativa. Para Almeida e Silva (2019), nos últimos anos houve a adoção de metodologias estratégicas mais incisivas e modificações curriculares mais expressivas para provocar mudanças na dinâmica de trabalho interprofissional, entretanto, ainda se encontra dificuldades em relacionar o visto em formação e o suprimento da necessidade do sistema de saúde no trabalho colaborativo.

Assim, acredita-se que a adoção de políticas que fortaleçam a EIP possa trazer transformações para as práticas de saúde, principalmente na integração e colaboração entre os profissionais, com foco nas necessidades de saúde dos usuários e população, assegurando maior segurança à assistência do cuidado, redução de erros dos profissionais de saúde e de custos do 
sistema de saúde, contribuindo, dessa forma, para um SUS forte e capaz de dar respostas aos problemas e necessidades de saúde da população brasileira.

Costa et al. (2015) compreendem a EIP como uma potente abordagem para promover mudanças tanto na atenção à saúde nos serviços, quanto na formação profissional em saúde. Para os autores, os princípios da EIP "se articulam com os propósitos de se avançar na perspectiva da integralidade da atenção e fortalecer a lógica do trabalho em equipe centrado nas necessidades dos usuários, em suas dimensões individual e coletiva".

Alguns pontos devem ser destacados, o planejamento das atividades não envolveu os professores e escolares, e devido ao restrito número de dias disponibilizados e ao elevado número de turmas para a atividade não foi possível envolver nas dinâmicas todos os funcionários da escola como merendeiros e auxiliares de serviços gerais, assim como os pais ou responsáveis. Sugere-se o envolvimento de toda comunidade escolar nas atividades educativas, para dessa forma, promover a articulação de saberes por meio da participação de todos os atores envolvidos (Ministério da Saúde, 2011).

\section{Considerações Finais}

A experiência vivenciada no planejamento e implementação da ação educativa desenvolvida junto aos escolares oportunizou aos docentes, discentes e profissionais de saúde vivenciar a prática interprofissional como uma forma de incorporar um dos princípios do SUS, a integralidade do cuidado. Visto que "a integralidade se constrói na práxis do conjunto dos profissionais dos serviços de saúde e nas diferentes formas de encontro desses profissionais com e no serviço" (SILVA e SENA, 2008, p.49).

Refletiu-se que a valorização do trabalho em equipe possibilita a construção de conhecimento de diferentes saberes e interação entre os atores do PET-Saúde/Interprofissionalidade, possibilitando vivenciar um dos lemas da EIP "aprender juntos para trabalhar juntos por meio de práticas colaborativas em saúde".

Espera-se que este trabalho motive equipes da Estratégia de Saúde da Família em implementar e publicar ações educativas através do Programa Saúde na Escola, considerando a potencialidade inteprofissional disponível na atenção básica e incorporando metodologias ativas em consonância com o contexto dos escolares.

\section{Referências}

Almeida, R. G., Teston, E. F., \& Medeiros, A. A. (2019). A interface entre o PET-Saúde/Interprofissionalidade e a Política Nacional de Educação Permanente em Saúde. Saúde Debate, 43(1), 97-105.

Barr, H. (1998). Competent to collaborate, towards a competency-based model for interprofessional education. J Interprofessional Care, 12 (2), $181-188$.

Barr, H. (2005). Interprofessional education: today, yesterday and tomorrow: a review. Health Sciences and Practice Network.

Barros, A. J. P., \& Lehfeld, N. A. S. (2000). Projeto de Pesquisa: propostas metodológicas. Vozes.

Batista, N. A. (2012). Educação Interprofissional em Saúde:Concepções e Práticas. Caderno FNEPAS, (2), 25-28.

Berbel, N. A. N. (2011). As metodologias ativas e a promoção da autonomia de estudantes. Ciências Sociais e Humanas, 32 (1), $25-40$.

Costa, M. V. (2016). The interprofessional education in Brazilian context: some reflections. Interface, 20(56), 197-198.Silva, K. L., Sena, R. R. (2008). Integralidade do cuidado na saúde: indicações a partir da formação do enfermeiro. Rev Esc Enferm USP, 42(1), 48-56

Costa, M. V., Patrício, K. P., Câmara, A. M., Azevedo, G. D., \& Batista, S. H. (2015). Pro-Health and PET-Health as interprofessional education spaces. Interface, 19(1), 709-720.

Ellery, A. E. (2014). Interprofissionalidade na Estratégia Saúde da Família: condições de possibilidade para a integração de saberes e a colaboração interprofissional. Interface, 18(48), 213-215.

Ellery, A. E., Pontes, R. J., \& Loiola, F. A. (2013). Campo comum de atuação dos profissionais da Estratégia Saúde da Família no Brasil: um cenário em construção. Physis Revista de Saúde Coletiva, 23(2), 415-437.

França, T., Magnago, C., Santos, M., Belisário, S., \& Silva, C. (2018). PET-Saúde/GraduaSUS: retrospectiva, diferenciais e panorama de distribuição dos projetos. Saúde Debate, 42(2), 286-301. 
Research, Society and Development, v. 10, n. 3, e52410313628, 2021

(CC BY 4.0) | ISSN 2525-3409 | DOI: http://dx.doi.org/10.33448/rsd-v10i3.13628

Interprofessional Education Collaborative Expert Panel. (2011). Core competencies for interprofessional collaborative practice: Report of an expert panel. Washington, D.C.: Interprofessional Education Collaborative.

Minayo, M. C. (2012). Pesquisa Social: teoria, método e criatividade. Vozes.

Ministério da Saúde (2011). Instrutivo Programa Saúde na Escola (PSE).

Peduzzi, M., \& Agreli, H. (2018). Teamwork and collaborative practice in Primary Health Care. Interface, 22 (2), $1525-1534$.

Peduzzi, M., Norman, I., Germani, A., Silva, J., \& Souza, G. (2013). Interprofessional education: training for healthcare professionals for teamwork focusing on users. Rev. Esc. Enferm. USP,47(4), 977-983.

Reeves, S. (2016). Porque precisamos da educação interprofissional para um cuidado efetivo e seguro. Interface, $20(56), 185-96$.

Santos, C. A. (2005). Construção Social do Conceito de Identidade Profissional. Interações: Sociedade e as novas modernidades, 5(8). 